Revista Internacional de Apoyo a la Inclusión, Logopedia, Sociedad y Multiculturalidad.

Volumen 7, Número 2, junio 2021, ISSN: 2387-0907. DOI: https://dx.doi.org/10.17561/riai.v7.n2.6363

\title{
Metodologías inclusivas y emergentes para la formación docente en inclusión educativa.
}

(Inclusive and emerging methodologies to tecaher training in educational inclusion.)

\author{
María Isabel Quesada López \\ Universidad de Jaén (España) \\ miq10002@ujaen.es
}

Fecha recepción: 08-04-2021

Páginas 110-117

Fecha aceptación: 25-05-2021

\section{Resumen.}

Este artículo procura clarificar el concepto de inclusión educativa como el derecho de todas las personas a una educación de calidad, con la finalidade de reducir y eliminar la exclusión de cualquier tipo. Asimismo, se pretende oferecer distintas metodologías y estrategias inclusivas que pueden ser aplicadas en el aula para favorecer la inclusión del alumnado y mejorar el proceso educativo. Del mismo modo, la educación del siglo XXI debe de estar a la altura de la sociedad actual, por lo que se presentan distintas metodologías emergentes en educación que deberían implementarse en los centros educativos durante los próximos años, ya que nos encontramos en la era de la Tecnología de la Comunicación y la Información, y esta ha de estar presente en el proceso de enseñanza y aprendizaje del alumnado. Resulta imprescincible que los docentes estén en formación continua y permanente y se encuentren preparados y formados para aplicar dichas metodologías y enfrentarse a los nuevos retos.

Palabras clave: metodologías inclusivas; metodologías emergentes; formación docente; inclusión educativa; exclusión

\begin{abstract}
.
This article aims to give clarify to the concept of educational inclusion as the right of all people to a quality education, with the aim of reducing and eliminating exclusion of any kind. Furthermore, it is intended to offer different inclusive methodologies and strategies which can be applied in the classroom to favor the inclusion of students and improve the educational process. In the same way, education in the 21 st century must be at the level of today's society, which is why different emerging methodologies in education are presented that should be implemented in educational centers during the coming years, due to the fact that we are in the era of Communication and Information Technology, and this must be present in the teaching and learning process of students. It is essential that teachers are in continuous and permanent training and are prepared and trained to apply these methodologies and face new challenges.
\end{abstract}

Keywords: inclusive methodologies; emerging methodologies; teacher training; educational inclusion; exclusion 
Revista Internacional de Apoyo a la Inclusión, Logopedia, Sociedad y Multiculturalidad.

Volumen 7, Número 2, junio 2021, ISSN: 2387-0907. DOI: https://dx.doi.org/10.17561/riai.v7.n2.6363

\section{1.-Introducción.}

El derecho a la educación y el objetivo de Desarrollo Sostenible son unos de los principios primordiales de la comunidad (UNESCO, 2010). Debido a distintos factores, ya sean económicos, sociales o culturales, continúan existiendo personas que no logran alcanzar dicho derecho. Sin embargo, la lucha es constante para que todos los individuos puedan formar parte de una educación de calidad basada en la igualdad de oportunidades.

Según Escudero y Martínez (2011), la inclusión, hoy día, ha tomado protagonismo, con la finalidad de valorar la diversidad como enriquecedora y beneficiosa para todos los seres humanos. Para lograr una inclusión educativa es, por tanto, imprescindible desarrollar prácticas inclusivas que favorezcan la participación del alumnado y disminuyan la exclusión. No obstante, el mundo actual de las "Tecnologías de la Información y la Comunicación" demandan un nuevo desafío. Según Hurtado et al. (2019), el papel del docente, en este desafío emergente, es clave para su consecución, pues es la persona encargada de dar la respuesta educativa más apropiada al alumnado. La formación del profesorado presenta una gran responsabilidad, puesto que debe desarrollarse de manera profesional para atender las necesidades del alumnado, desde una sociedad en constante cambio. Dicha formación debe ser continua y permanente, a partir de una visión inclusiva y de la búsqueda de herramientas y estrategias adecuadas y no usuales en su práctica docente, que favorezcan la calidad educativa de todas y todos los estudiantes.

Por lo tanto, este artículo realiza una revisión de los conceptos de inclusión educativa y formación docente, junto a la descripción y propuesta de distintas metodologías inclusivas y emergentes, con la finalidad de ser conocidas por el profesorado e implementadas en su formación para lograr una verdadera inclusión en la educación.

\section{2.-Marco teórico.}

\section{1.-Inclusión Educativa.}

La inclusión educativa, según Booth y Ainscow (2000), requiere de un conjunto de prácticas y procesos que reduzcan la exclusión del alumnado y aumenten su participación en la cultura, el currículo y en las comunidades de aprendizaje. El término de inclusión hace referencia a la participación en la educación de todos aquellos discentes que se encuentran en riesgo de exclusión. Para que se dé una verdadera inclusión hay que procurar la eliminación o reducción de las barreras de cualquier tipo, de manera que los estudiantes puedan acceder e intervenir en la vida educativa. Por otro lado, esta concepción destaca por no percibir la diversidad como un inconveniente que ha de ser resuelto, sino como una oportunidad de mejora y una manera para enriquecer el aprendizaje de los demás. Hace referencia a una coordinación y apoyo entre los centros educativos y la comunidad, el entorno, puesto que la inclusión educativa forma parte de la consecución de la inclusión social. 
Los autores definen distintas dimensiones para que exista una inclusión de calidad en educación. En primer lugar, ha de crearse una cultura inclusiva, asociada a crear una comunidad educativa con un ambiente colaborador, acogedor y seguro, para que los estudiantes alcancen altos logros. En segundo lugar, se encuentra la dimensión que hace referencia a la elaboración de políticas inclusivas, las que han de agruparse en un marco único que favorezca y permita la participación y el aprendizaje de todos los estudiantes. Por último, el desarrollo de prácticas inclusivas. Esta dimensión implica el reflejo de la cultura y la política inclusiva en la educación. La implementación de prácticas inclusivas tiene que ir ligado a una seguridad de que las actividades tanto dentro del aula como fuera de ella, faciliten la participación de todos los estudiantes, teniendo en cuenta el contexto en el que cada uno se encuentra.

Desde la perspectiva de García et al. (2013), la educación inclusiva consiste principalmente en ofrecer a todos y cada uno de los estudiantes gran calidad educativa, indistintamente de las condiciones que presente, ya sean sociales, culturales, personales, etc. Este concepto ha evolucionado a lo largo del tiempo y continúa siendo un reto al que tienen que afrontarse los distintos sistemas educativos. Aunque este tipo de educación parta del objetivo de que todos los niños y niñas tienen derecho a la educación y a que esta sea de calidad, vemos cómo esto dependerá de los recursos invertidos en educación, formación del profesorado, importancia de la educación especial, cooperación de la familia, es decir, del contexto que se dé.

\section{2.-Formación docente.}

Según Marcelo (1994), la formación docente hace referencia a la educación que reciben aquellos que van a ser profesionales de la enseñanza. Este concepto hace referencia a la interacción entre un formador y un formado, en la cual se intercambian una serie de conocimientos en los que ambos están interesados y comprometidos, para la consecución de una serie de objetivos que cumplir. En la formación docente, conviven dos tipos de formaciones, la académica y la pedagógica, puesto que deben aprender a enseñar. Se habla de formar a formadores, por lo que hay que tener en cuenta la repercusión que tiene la acción docente. La calidad de la enseñanza y de la educación en general, depende de la formación que haya recibido el profesorado. La didáctica adquiere un papel fundamental en este campo educativo, ya que es la base de las metodologías y estrategias de enseñanza que denotarán el quehacer educativo del docente, así como el papel que este desempeñará, por ejemplo, instructor, facilitador del aprendizaje, guía, etc.

Este autor alude a una serie de distinciones sobre las fases de la formación docente:

- "Fase pre-entrenamiento": se refiere a las vivencias y experiencias personales referentes a la enseñanza que posee el futuro aspirante a docente.

- "Fase de formación inicial": es la etapa en la que el futuro docente ser forma en pedagogía y otras materias académicas, así como la realización de prácticas para la enseñanza. 
Revista Internacional de Apoyo a la Inclusión, Logopedia, Sociedad y Multiculturalidad.

Volumen 7, Número 2, junio 2021, ISSN: 2387-0907. DOI: https://dx.doi.org/10.17561/riai.v7.n2.6363

- "Fase de iniciación": fase en la que se ejerce de docente de manera primeriza, aprendiendo de la práctica y a través de la experiencia.

- "Fase de formación permanente": el docente propiamente dicho, continua con su formación para mejorar su práctica y adquirir nuevas estrategias y destrezas.

La formación permanente y continua del profesorado es fundamental, debido al papel que este ejerce en la educación junto a su influencia social. Actualmente, está cobrando un importante papel en las distintas investigaciones, puesto que se declara que el profesorado debe de estar capacitado para desarrollar su práctica en distintos contextos. La diversidad del alumnado es cada vez más patente, lo que requiere docentes formados en atender dicha diversidad, un completo reto para ellos. A pesar de la diversidad, las metodologías y estrategias en las que se forma el profesorado están destinadas a estudiantes homogéneos. La muestra de ello son los programas de educación del profesorado. Ante el desafío anteriormente citado, se hace imprescindible la formación del profesorado en inclusión, mediante la cual los profesionales puedan adquirir nuevas herramientas que destruyan barreras de aprendizaje y proporcionen una calidad educativa. Asimismo, cabe destacar que los docentes han de estar en formación permanente para renovar su práctica, adecuándose a las necesidades del nuevo alumnado y a los avances sociales, culturales y tecnológicos de la época. (Infante, 2010).

\section{3.-Metodologías inclusivas.}

Para conseguir una educación verdaderamente inclusiva en el aula, la escuela, los docentes y toda la comunidad educativa han de estar comprometidos para alcanzar dicha inclusión. Para ello se desarrollan diferentes estrategias entre las que cabe destacar las prácticas inclusivas. "Se entiende por estrategias o prácticas educativas inclusivas aquel tipo de estructuras, tareas $y / 0$ actividades que ofrecen oportunidades reales de aprender a todo el alumnado" (Chiner Sanz, 2011, p. 95).

A continuación, se describen distintas metodologías inclusivas para trabajar en el aula, según Moliner et al. (2017):

Estrategias de aprendizaje cooperativo. El aprendizaje cooperativo es una estrategia grupal, que consiste en cooperar para conseguir objetivos que son beneficiosos tanto para uno mismo como para todos los integrantes del equipo. Los grupos suelen ser heterogéneos para compensar capacidades y limitaciones y entre todos aporten diversas características en el trabajo. Algunas estrategias para trabajar el aprendizaje cooperativo son:

- TAI («Team Assisted Individualization»): el grupo trabaja los mismos contenidos, pero posee distintos objetivos 0 actividades que son atribuidas de manera individualizada y adaptadas según las necesidades de cada uno de ellos. Cada uno posee responsabilidad individual en su tarea, aunque debe apoyar al resto. 
Revista Internacional de Apoyo a la Inclusión, Logopedia, Sociedad y Multiculturalidad.

Volumen 7, Número 2, junio 2021, ISSN: 2387-0907. DOI: https://dx.doi.org/10.17561/riai.v7.n2.6363

- Tutoría entre iguales: en esta técnica se produce una interacción entre dos estudiantes. Uno de ellos asume el rol de enseñar y otro de ser enseñado. De esta forma, ambos se benefician de forma recíproca.

Estrategias de aprendizaje dialógico. Esta estrategia, a través del diálogo, favorece las interacciones entre el alumnado, sin existir relaciones jerárquicas entre ellos. Asimismo, a través del diálogo y la reflexión, resulta imprescindible que el alumnado adquiera los conocimientos de manera que se produzca un aprendizaje significativo. Para llevar a cabo dicha estrategia se utilizan diversas metodologías:

- Grupos interactivos: la presente metodología consiste en aumentar la interacción y la participación de los estudiantes, de manera que el aprendizaje sea constante. Los grupos trabajan las diferentes actividades propuestas, intercambiándolas entre ellos para que todos las realicen.

- Tertulias dialógicas: esta estrategia permite al estudiantado construir el conocimiento mediante el diálogo en la tertulia. A través de la lectura, primero individual y luego grupal, se reflexiona sobre distintos aspectos.

Estrategias de aprendizaje servicio y comunitario. Fundamenta su aprendizaje, a través de la participación activa y el servicio a la comunidad. Esta estrategia resulta muy dinámica, pues el proceso de enseñanza-aprendizaje es muy práctico y experimental. Esto hace que el alumnado vivencie su propio aprendizaje, lo que favorece la motivación y hace que este sea más significativo. La presente estrategia precisa de um gran compromiso de los discentes.

Estrategias para fomentar la participación social. Dicha metodología se utiliza cuando hemos divido la clase en equipos e incorporamos al grupo, alumnado con NEAE 0 incluso estudiantes de nuevo ingreso al aula o centro educativo. El grupo le enseñará diversas pautas de las tareas que ya estarán establecidas en el ámbito diario de trabajo del aula. A continuación, se describe una serie de metodologías que son utilizadas para llevar a cabo esta estrategia:

- Sistema de compañeros y amigos: esta estrategia involucra a otros compañeros que son voluntarios para ayudar a aquellos alumnos que necesitan ayuda social tanto en situaciones escolares como extraescolares.

- Círculo de amigos: esta técnica consiste en realizar varios círculos: para los más allegados y amigos cercanos, las relaciones lejanas y las estrictamente profesionales. Seguidamente, se reflexiona sobre la situación del alumnado con necesidad y se busca establecerlo en el círculo que se pretenda.

- Comisiones de apoyo entre compañeros: la metodología tratada consiste en la creación de comisiones de compañeros en el aula, y poco a poco se van uniendo por turnos distintos alumnos. Esto favorece la ayuda y el apoyo entre los compañeros del grupo-clase.

- Alumno ayudante: el grupo-clase interactúa sobre qué alumnado del aula posee ciertas características o cualidades, que sean adecuadas para ayudar y acompañar a aquellos discentes que requieren dicho apoyo. 
Estrategias integrales. Esta metodología se emplea para la atención a la diversidad en el aula, donde se dan distintas estrategias de manera simultánea. No solo abarca la modificación de espacios, tiempos, grupos, sino que se basa en el conjunto de todas ellas. Seguidamente, se describen algunos ejemplos de estrategias integrales:

- Rincones y talleres: esta estrategia destruye la idea de que todo el alumnado deba realizar y compartir la misma tarea. Gracias a ellos, se respetan las capacidades y limitaciones del estudiantado, así como el ritmo individual de trabajo de cada uno de ellos.

- Proyectos de trabajo inclusivos: esta idea de proyectos inclusivos solo puede lograrse a través de una escuela abierta, un cirriculum flexible y un compromiso docente. La atención a la diversidad debe ser diaria y continua, no debe quedar trabajos de días señalados y organizados.

- Enseñanza compartida: dicha estrategia aboga la importancia de poseer dos docentes en una misma aula. Este hecho supone la mejora de la comunicación y la acción mutua entre todo el grupo-clase.

\section{4.-Metodologías emergentes.}

La actualidad exige una modificación urgente en lo que a métodos de enseñanza se refiere. El alumnado convive en el mundo de la "tecnología de la información y la comunicación" y, la educación no puede no acercarse a ello. Hoy en día, los discentes exigen ser los protagonistas de su propio aprendizaje, lo que implica el uso de metodologías emergentes que favorezcan la participación activa y conecten con la realidad de los estudiantes. Asimismo, se reclama el desarrollo de la creatividad y la acción de emprender y, gracias a la tecnología emergente, esto puede lograrse con mayor facilidad. La implementación de estas metodologías en las aulas, suponen grandes desafíos para el personal docente, que ha de estar preparado y formado para ello. Del mismo modo, del Águila (2019), indica que, para lograr un aprendizaje real y significativo, se pueden llevar a la práctica metodologías como la gamificación, aula invertida, realidad aumentada, aprender a emprender, rutinas de pensamiento, entre otra gran variedad. No obstante, se describen algunas de las metodologías más emergentes, que aún supone un reto implementar en las aulas:

Realidad aumentada. Según Díaz (2017), gracias a la realidad virtual, la realidad aumentada tomó protagonismo en el ámbito social y educativo. No obstante, la implementación en las aulas de esta tecnología emergente es aún un poco atrevida. La realidad aumentada se trata de una metodología activa y dinámica, que conecta el mundo real con una serie de contenidos didácticos que se pretenden trabajar. Además de favorecer la motivación y la implicación en el aprendizaje, esta estrategia también mejora el desarrollo cognitivo, las habilidades visoespaciales, la atención, entre otras muchas competencias.

Desing Thinking. Arias et al. (2019), describen esta metodología como un proceso creativo en el que el alumnado puede experimentar o incluso crear y diseñar ideas originales y flexibles. Debido a las posibilidades que ofrece esta estrategia, pueden analizarse multitud de problemas, gracias a los cuales se oferta la oportunidad de 
proporcionar distintos tipos de soluciones. Dentro de esta metodología se encuentran una serie de partes a seguir: empatía, definir, idear, prototipar y evaluar.

Flipped Classroom. Según Berenger (2016), esta metodología modifica la forma de enseñanza-aprendizaje tradicional, puesto que se alteran los roles comúnmente adoptados. Está diseñada para que el alumnado trabaje los contenidos, generalmente en casa, y realicen actividades o proyectos en el aula. De este modo, queda explicado como su propio nombre indica, aula invertida, pues lo que normalmente se enseñaría en el aula, se realiza fuera de ella, y los deberes o tareas que se realizan en casa, se hacen en clase. Esta estrategia favorece el trabajo autónomo del alumnado y aumenta su motivación e interés hacia el aprendizaje. El docente facilita al alumnado la información teórica de los contenidos que se pretendan trabajar, de una manera más atractiva y dinámica, por ejemplo, vídeos, podcasts, power points, etc. Mobile learning. Mora (2013), define esta estrategia como una nueva manera de aprendizaje a través de dispositivos móviles, como un teléfono, una tableta, consola, entre otros. La emergencia de la tecnología, así como el uso habitual que se hace de ella, no puede quedarse atrás en el sistema educativo. Asimismo, esta metodología hace que el aprendizaje esté siempre presente, debido a que puede trasladarse desde el aula a cualquier punto, gracias a la virtualidad. También se ven ampliadas las posibilidades de interacción, ya que desde estos dispositivos pueden tratarse los contenidos, actividades y tareas a trabajar, comunicación a través de foros, blogs, y otras muchas posibilidades.

\section{3.-Conclusión.}

La inclusión educativa es imprescindible para lograr um modelo educativo ideal y de calidad. Todos los alumnos tienen derecho a la educación y han de partir en igualdad de oportunidades, ofreciendo al alumnado aquello que mejor se ajuste a sus necesidades. Para ello, el professorado ha de estar em formación permanente para mejorar su práctica y poder ofrecer un abanico de metodologías inclusivas e innovadoras. Además, teniendo en cuenta la necesidad de trabajar en una sociedad donde la inclusión y la tecnologia son fundamentales, resulta imprescindible conocer distintas metodologías inclusivas, así como metodologías emergentes, para poder aplicarlas en el aula y estar a la altura de la educación en la que vivimos hoy en día.

Conocer las distintas metodologías inclusivas y emergentes suponen um reto tanto para la sociedad como para los docentes, sin embargo es necesario para lo que verdaderamente importa, que la educación del alumnado sea óptima.

\section{4.-Referencias.}

Arias, H., Jadán, J. y Gómez, L. (2019). Innovación Educativa en el aula mediante DesignThinking y Game Thinking. Revista cuatrimestral de divulgación científica, 6(1),82-95. https://doi.org/10.21503/hamu.v6i1.1576

Berenguer, C. (2016). Acerca de la utilidad del aula invertida o flipped classroom. XIV Jornadas de redes de investigación en docencia universitaria. 
Revista Internacional de Apoyo a la Inclusión, Logopedia, Sociedad y Multiculturalidad.

Volumen 7, Número 2, junio 2021, ISSN: 2387-0907. DOI: https://dx.doi.org/10.17561/riai.v7.n2.6363

Investigación, innovación y enseñanza universitaria: enfoques pluridisciplinares. 1466- 1480.

Booth, T., Ainscow, M., Black-Hawkins, K., Vaughan, M., \& Shaw, L. (2000). Índice de inclusión. Centro de los Studios en la Educacion.

Chiner Sanz, E. (2011). Las percepciones y actitudes del profesorado hacia la inclusión del alumnado con necesidades educativas especiales como indicadores del uso deprácticas educativas inclusivas en el aula [Tesis de doctorado no publicada, Universidad de Alicante]. http://rua.ua.es/dspace/handle/10045/19467

Del Águila Ríos, Y., Capelo, M., Varela, J., Antequera, J. y Barroso, J. (2019). Creatividad y tecnologías emergentes en educación. Revista INFAD de Psicología. International Journal of Developmental and Educational Psychology, 3(1), 527-534. https://doi.org/10.17060/ijodaep.2019.n1.v3.1529

Díaz, M. (2017). La emergencia de la Realidad Aumentada en la educación. Revista de Educación Mediática y TIC, 6(1), 1-3. https://doi.org/10.21071/edmetic.v6i1.5804

Escudero, J.M. y Martínez, B. (2011). Educación Inclusiva y cambio escolar. Revista Iberoamericana de Educación, 55, 85-105. https://doi.org/10.35362/rie550526

García, I., Romero, S., Aguilar, C.L., Lomeli, K.A., y Rodríguez, D.C. (2013). Terminología internacional sobre la educación inclusiva. Actualidades Investigativas en Educación, 13(1), 182-211.

Infante, M. (2010). Desafíos a la formación docente: inclusión educativa. Estudios pedagógicos (Valdivia), 36(1), 287-297. https://doi.org/10.4067/S0718$\underline{07052010000100016}$

Marcelo, C. (1994). Formación del profesorado para el cambio educativo. Barcelona:PPU.

Moliner García, O., Sanahuja Ribés, A. y Benet Gil, A. (2017). Prácticas inclusivas en elaula desde la investigación-acción [Tesis de maestría no publicada, Universitat Jaume I]. http://repositori.uji.es/xmlui/handle/10234/168902 https://doi.org/10.6035/Sapientia127

Mora, F. (2013). El mobile learning y algunos de sus beneficios. Revista Calidad en la Educación Superior. 4(1), 47-67. https://doi.org/10.22458/caes.v4i1.453

Organización de las Naciones Unidas para la Educación, la Ciencia y la Cultura. (19 de abril de 2010). El derecho a la Educación. https://es.unesco.org/themes/derecho-a-educacion 